\title{
SYMPLECTIC MODELS FOR GENERAL INSERTION DEVICES *
}

\author{
$\mathrm{Y} . \mathrm{Wu}^{\dagger}$, E. Forest ${ }^{\ddagger}$, D. S. Robin, H. Nishimura, A. Wolski, LBNL, Berkeley, CA 94720, USA \\ V. N. Litvinenko, FEL Lab, Duke University, Durham, NC 27708, USA
}

\begin{abstract}
A variety of insertion devices (IDs), wigglers and undulators, linearly or elliptically polarized, are widely used as high brightness radiation sources at the modern light source rings. Long and high-field wigglers have also been proposed as the main source of radiation damping at next generation damping rings. As a result, it becomes increasingly important to understand the impact of IDs on the charged particle dynamics in the storage ring. In this paper, we report our recent development of a general explicit symplectic model for IDs with the paraxial ray approximation. High-order explicit symplectic integrators are developed to study real-world insertion devices with a number of wiggler harmonics and arbitrary polarizations.
\end{abstract}

\section{INTRODUCTION}

In the storage ring, symplectic integration provides an essential tool to study the long-term behavior of the single particle dynamics. Magnetic multipole elements, such as quadrupoles and sextupoles, are modeled using a so-called impulse boundary approximation, in which the magnetic field is assumed to be constant ( $s$-independent) within the effective boundary of the magnet and zero outside. Such a magnetic field model allows one to use a special vector potential, $\vec{A}=A_{z}(x, y) \hat{z}$ for each magnet. As a result, the charged particle Hamiltonian can be separated into the drift-kick combination of Ruth [1]: $H=T(\vec{p})+V(\vec{q})$, where $T(\vec{p})$ is a drift, $V(\vec{q})$ is a kick. A second order Lie map approximation can be constructed for this type of Hamiltonians [2], resulting in an explicit symplectic integrator for magnetic multipoles. Implementing explicit multipole integration schemes in various tracking codes in 1990 's, it became possible to compute the charged particle trajectories after a large number of turns without introducing artificial damping or anti-damping. These tracking codes have become critical tools for designing the third generation light storage rings with small emittance as well as high energy physics collider rings with high luminosity.

The Ruth's integrator was extended by Forest [3] to Hamiltonians which can be separated into a multiple number of integral parts containing coordinates and momenta belonging to different canonical pairs [3]. This generalized technique can be used to construct symplectic integrators for planar wigglers with an infinite pole width. However, this method is limited to Hamiltonians with a two-

\footnotetext{
* Work supported by the Director, Office of Energy Research, Office of Basic Energy Sciences, Material Sciences Division, U.S. Department of Energy, under Contract No. DE-AC03-76SF00098

†ywu@lbl.gov

$\ddagger$ Also at KEK, 1-1 Oho, Tsukuba, Ibaraki 305-0810, Japan
}

dimensional magnetic field.

With their increased use in modern light source rings and next generation damping rings, insertion devices (IDs) with arbitrary polarizations and rich harmonic contents become critical components of these rings. It has become essential to understand and minimize the impact of IDs on the charged particle beam dynamics. This is particularly true for rings in which the nonlinear dynamics are dominated by wigglers. Until recently, the most comprehensive wiggler modeling was performed by the BESSY group using the generating function based implicit method [4], [5]. In this method, a symplectic higher order map was produced numerically for the insertion device. However, besides the convergence issues and limited order of the map which can be produced, the implicit method has difficulties in dealing with parameter-dependency of the field, and is limited to producing maps for a given design orbit.

In this paper, we report the development of a general explicit symplectic model for insertion devices such as wigglers and undulators. This model applies a recently developed symplectic integration method for a 3D magnetic field Hamiltonian with the paraxial-ray approximation [6]. The importance of this method is that it allows the generation of canonical maps for an ID with any parameter dependency by tracking through the ID once with a differential algebra package. More importantly, since the method is explicit in nature, direct trajectory tracking in the real magnetic field can be performed for dynamic aperture studies. Consequently, this provides a benchmark for the dynamics studies in which the large amplitude motion may or may not be properly described by the on-axis map of a given order.

\section{EXPLICIT INTEGRATOR FOR 3D MAGNETIC FIELD}

In this section, we will briefly outline the method to construct a second order (and higher) explicit symplectic integrator for a 3D magnetic field with $s$-dependency. The details of this technique can be found in [6]. Let us start with a charged particle Hamiltonian with a varying magnetic field along $z$ in the Cartesian coordinate system:

$$
\begin{aligned}
& H\left(x, p_{x}, y, p_{y}, \delta, l ; z\right) \\
& =-\sqrt{(1+\delta)^{2}-\left(p_{x}-a_{x}\right)^{2}-\left(p_{y}-a_{y}\right)^{2}}-a_{z} \\
& \approx-\delta+\frac{\left(p_{x}-a_{x}\right)^{2}}{2(1+\delta)}+\frac{\left(p_{y}-a_{y}\right)^{2}}{2(1+\delta)}-a_{z}
\end{aligned}
$$

where $p_{x, y}=P_{x, y} / P_{0}$ is the normalized transverse momenta, $\delta=P / P_{0}-1$ is the relative momentum deviation, $l$ is the path length, $a_{x, y, z}(x, y, z)=q A_{x, y, z}(x, y, z) /\left(P_{0} c\right)$ 
is the normalized vector potential. A paraxial-ray approximation is made for the Hamiltonian, which is valid and widely used for large rings.

To see that explicit symplectic integration is possible for this Hamiltonian, we should extend our phase space to include $\left(z, p_{z}\right)$ as the 4 th dimension [7]. The resulting equivalent Hamiltonian in the 4D space is:

$$
\begin{aligned}
& K\left(x, p_{x}, y, p_{y}, \delta, l, z, p_{z} ; \sigma\right) \\
& \approx-\delta+\frac{\left(p_{x}-a_{x}\right)^{2}}{2(1+\delta)}+\frac{\left(p_{y}-a_{y}\right)^{2}}{2(1+\delta)}-a_{z}+p_{z},
\end{aligned}
$$

where $\sigma$ is the new independent variable and $d z=d \sigma$. Noticing that the new Hamiltonian $K$ does not depend on $\sigma$ explicitly, for a given integration step size, $\Delta \sigma$, we can write down the Lie map solution symbolically as:

$$
\mathcal{M}(\Delta \sigma)=\exp (:-\Delta \sigma K:)
$$

Next, we split the Hamiltonian, $K$, into four parts, $K=$ $K_{1}+K_{2}+K_{3}+K_{4}$, and

$$
\begin{aligned}
K_{1} & =p_{z} \\
K_{2} & =a_{z} \\
K_{3} & =\frac{\left(p_{y}-a_{y}\right)^{2}}{2(1+\delta)} \\
K_{4} & =-\delta+\frac{\left(p_{x}-a_{x}\right)^{2}}{2(1+\delta)}, \\
\mathcal{N}_{i}(\Delta \sigma) & =\exp \left(:-\Delta \sigma K_{i}:\right) .
\end{aligned}
$$

Using these partial maps, we can readily write down a second order approximation for the map $\mathcal{M}$ :

$$
\begin{aligned}
\mathcal{M}_{2}(\Delta \sigma)= & \mathcal{N}_{1}\left(\frac{\Delta \sigma}{2}\right) \mathcal{N}_{2}\left(\frac{\Delta \sigma}{2}\right) \mathcal{N}_{3}\left(\frac{\Delta \sigma}{2}\right) \\
& \mathcal{N}_{4}(\Delta \sigma) \mathcal{N}_{3}\left(\frac{\Delta \sigma}{2}\right) \mathcal{N}_{2}\left(\frac{\Delta \sigma}{2}\right) \mathcal{N}_{1}\left(\frac{\Delta \sigma}{2}\right) \\
\approx & \mathcal{M}(\Delta \sigma)+O\left((\Delta \sigma)^{3}\right) .
\end{aligned}
$$

To see that maps $\mathcal{N}_{3}\left(\frac{\Delta \sigma}{2}\right)$ and $\mathcal{N}_{4}(\Delta \sigma)$ are also exactly solvable, we use the following generating functions to transfer Hamiltonians $K_{3}$ and $K_{4}$ to some new coordinate systems:

$$
\begin{aligned}
& K_{3}=\mathcal{A}_{y} \frac{p_{y}^{2}}{2(1+\delta)}, K_{4}=\mathcal{A}_{x}\left(-\delta+\frac{p_{x}^{2}}{2(1+\delta)}\right), \\
& \mathcal{A}_{y}=\exp \left(:-\int_{y_{0}}^{y} a_{y} d y:\right), \mathcal{A}_{x}=\exp \left(:-\int_{x_{0}}^{x} a_{x} d x:\right) .
\end{aligned}
$$

We observe that the operations by $\mathcal{A}_{x}$ and $\mathcal{A}_{y}$ on the phase space variables are explicit, consequently, $\mathcal{M}_{2}$ can be expressed as a product of a series of Lie maps which can be explicitly evaluated:

$$
\begin{aligned}
\mathcal{M}_{2}(\Delta \sigma)= & \exp \left(:-\frac{\Delta \sigma}{2} p_{z}:\right) \exp \left(:-\frac{\Delta \sigma}{2} a_{z}:\right) \\
& \left(\mathcal{A}_{y} \exp \left(:-\frac{\Delta \sigma}{2} \frac{p_{y}}{2(1+\delta)}:\right) \mathcal{A}_{y}{ }^{-1}\right)
\end{aligned}
$$


Similarly, we can construct an explicit integrator for planar vertical wigglers. By super-positioning vector potentials of a horizontal and a vertical wiggler with different field strengths, we are ready to model an arbitrarily polarized wiggler.

The harmonic content for a real wiggler magnet can be determined by using a two-dimensional discrete Fourier transformation after imposing the periodic condition in one of the transverse directions [9]. A large number of harmonics may be needed in order to achieve a high degree of accuracy because of the 3D nature of the field. However, it is expected that few modes are mainly responsible for the nonlinear beam dynamics. Therefore a reduced subset of modes can be used in modeling.

\section{WIGGLER FRINGE FIELDS}

The fringe effects can be treated separately as we do in the case of magnetic multipoles. A simple dipole hard edge model can be used for a planar wiggler with $\theta_{z n}=0$ in Eq. 8, where the magnetic field peaks at wiggler ends. As expected the linear effect is the edge focusing [7].

To properly treat the "real-world" wiggler fringe fields including the field tapering used for the orbit compensation, we need a symplectic model for such a field. Recognizing that the integration scheme outlined in section 2 is applicable to all types of 3D magnetic fields, we can readily apply this technique to the wiggler fringe fields.

First, we separate the wiggler magnetic field into "pure wiggler" field represented by the wiggler harmonics (see Eq. 8) and the fringe field. This fringe field peaks around the entrance and exit of the wiggler and tails off. Second, we need to choose a particular type of analytic representation for the fringe field. One of the candidates is the pseudo-multipoles, widely used in studying multipole fringe field [11]. The scalar potential is given by

$$
V(r, \phi, z)=\sum_{n=0} a_{n}(r, z) \sin (n \phi)+b_{n}(r, z) \cos (n \phi),
$$

where $a_{n}(r, z)=\sum_{k=0} c_{n, k}(z) r^{n+2 k}$ and $b_{n}(r, z)=$ $\sum_{k=0} d_{n, k}(z) r^{n+2 k}$, and $\vec{B}=-\nabla V$. Vanishing divergence for $\vec{B}$ requires $\nabla^{2} V(r, \phi, z)=0$, which yields the following recursive relation:

$$
\alpha_{n, k}(z)=-\frac{1}{4 k(n+k)} \frac{d^{2} \alpha_{n, k}}{d z^{2}},
$$

where $\alpha_{n, k}=c_{n, k}$ or $d_{n, k}$, and $k=1,2, \ldots$ To write down a corresponding vector potential of this field, we choose $\vec{A}=\left(A_{r}, A_{\phi}, A_{z}=0\right)$ :

$$
\begin{aligned}
A_{r}= & \sum_{n, k} n r^{n+2 k-1}\left\{\int_{z_{0}}^{z} d_{n, k}(z) d z \sin (n \phi)\right. \\
& \left.-\int_{z_{0}}^{z} c_{n, k}(z) d z \cos (n \phi)\right\} \\
A_{\phi}= & \sum_{n, k}(n+2 k) r^{n+2 k-1}\left\{\int_{z_{0}}^{z} d_{n, k}(z) d z \cos (n \phi)\right.
\end{aligned}
$$

$$
\left.+\int_{z_{0}}^{z} c_{n, k}(z) d z \sin (n \phi)\right\},
$$

where $z_{0}$ is an arbitrary location. And the corresponding vector potential in the Cartesian coordinate is given by:

$$
A_{x}=\cos \phi A_{r}-\sin \phi A_{\phi}, A_{y}=\sin \phi A_{r}+\cos \phi A_{\phi} .
$$

If $\vec{B}=\nabla \times \vec{A}$, truncating $k$ to a particular order for $\vec{A}$ would violate $\nabla \times \vec{B}=0$ at the higher orders, equivalent to artificially introducing a small current source term.

\section{SUMMARY}

In this paper, we have outlined a new technique to compute the motion of a charged particle in a three-dimensional magnetic field in an explicit symplectic manner under the paraxial-ray approximation. Applying this technique, we have developed explicit integrators for modeling general insertion devices in the storage ring. Multiple wiggler harmonics, arbitrary polarizations, real fringe fields of the IDs can all be treated properly in this model. We are in the process of incorporating this general insertion device model into traditional particle tracking codes, such as TRACY [10]. This will enable us to study the details of the single particle dynamics in the storage ring with insertion devices. Consequently, it will have a significant impact on the design of the next generation linear collider damping rings with damping wigglers, optimal use of insertion devices in the light source rings, and better understanding of the role that insertion devices play in the storage ring in general.

It is worth pointing out that the explicit symplectic technique developed here is not limited to insertion devices as illustrated by our treatment of the wiggler fringe field. Its applications extend to the studies the fringe field effects of super-conducing dipoles and wavelength shifters, final focus system with combined solenoids and quadrupoles, as well as the cross-talk effects of two closed placed magnetic devices.

\section{REFERENCES}

[1] R. D. Ruth, IEEE Trans. Nucl. Sci., ns-30, p.2669, 1983.

[2] E. Forest, R. D. Ruth, Physica D, vol. 43, p. 105, 1990.

[3] E. Forest, et al., Physics Letters A, 158, p. 99, 1991.

[4] J. Bahrdt, G. Wustefeld, BESSY TB Nr. 158 \& 163, 1991. M. Scheer, G. Wustefeld, Technischer Bericht TB N.169, 1992.

[5] M. Scheer and G. Wustefeld, Proc. PAC97, v.2, p2606, 1998.

[6] Y. Wu, E. Forest, D. S. Robin, LBNL-48172 (2001), to be published.

[7] E. Forest, "Beam Dynamics - A new attitude and framework", Harwood Academic, vol. 8, 1998.

[8] H. Yoshida, Phys. Lett. A, vol. 150, p. 262, 1990.

[9] A. Wolski, J. N. Corlett, Y. Wu, these proceedings.

[10] H. Nishimura, J. Bengtsson, LBNL, private communication.

[11] S. Caspi, et al., LBNL report, LBL-30313, UC-405 (1991). 